\title{
Towards an interoperability framework for observable property terminologies
}

\author{
Barbara Magagna ${ }^{1}$, Gwenaelle Moncoiffe ${ }^{2}$, Anusuriya Devaraju ${ }^{3}$, Pier Luigi Buttigieg ${ }^{4}$, Maria \\ Stoica ${ }^{5}$, and Sirko Schindler \\ ${ }^{1}$ Ecosystem Research and Environmental Information Management, Environment Agency Austria, Vienna, Austria \\ (barbara.magagna@umweltbundesamt.at) \\ ${ }^{2}$ National Oceanography Centre, British Oceanographic Data Centre, Liverpool, United Kingdom (gmon@bodc.ac.uk) \\ ${ }^{3}$ PANGAEA, University of Bremen, Bremen, Germany (adevaraju@marum.de) \\ ${ }^{4}$ Alfred-Wegener-Institut, Helmholtz-Zentrum für Polar- und Meeresforschung, Bremerhaven, Germany \\ (pier.buttigieg@awi.de) \\ ${ }^{5}$ University of Colorado, Boulder, United States (maria.stoica@colorado.edu) \\ ${ }^{6}$ Institute of Data Science, German Aerospace Center (DLR), Jena, Germany (sirko.schindler@dlr.de)
}

In October 2019, a new working group (InteroperAble Descriptions of Observable Property Terminology or I-ADOPT WG ${ }^{1}$ ) officially launched its 18-month workplan under the auspices of the Research Data Alliance (RDA) co-led by ENVRI-FAIR ${ }^{2}$ project members. The goal of the group is to develop a community-wide, consensus framework for representing observable properties and facilitating semantic mapping between disjoint terminologies used for data annotation. The group has been active for over two years and comprises research communities, data centers, and research infrastructures from environmental sciences. The WG members have been heavily involved in developing or applying terminologies to semantically enrich the descriptions of measured, observed, derived, or computed environmental data. They all recognize the need to enhance interoperability between their efforts through the WG's activities.

Ongoing activities of the WG include gathering user stories from research communities (Task 1), reviewing related terminologies and current annotation practices (Task 2) and - based on this defining and iteratively refining requirements for a community-wide semantic interoperability framework (Task 3). Much like a generic blueprint, this framework will be a basis upon which terminology developers can formulate local design patterns while at the same time remaining globally aligned. This framework will assist interoperability between machine-actionable complex property descriptions observed across the environmental sciences, including Earth, space, and biodiversity science. The WG will seek to synthesize well-adopted but still disparate approaches into global best practice recommendations for improved alignment. Furthermore, the framework will help mediate between generic observation standards $\left({\mathrm{O} \& \mathrm{M}^{3}}^{3}, \mathrm{SSNO}^{4}\right.$, SensorML $\left.{ }^{5}, \mathrm{OBOE}^{6}, ..\right)$ and current community-led terminologies and annotation practices, fostering harmonized implementations of observable property descriptions. Altogether, the WG's work will boost the Interoperability component of the FAIR principles (especially principle 13) by encouraging convergence and by enriching the terminologies with qualified references to other resources. We envisage that this will greatly enhance the global effectiveness and scope of tools operating across 
terminologies. The WG will thus strengthen existing collaborations and build new connections between terminology developers and providers, disciplinary experts, and representatives of scientific data user groups.

In this presentation, we introduce the working group to the EGU community, and invite them to join our efforts. We report the methodology applied, the results from our first three tasks and the first deliverable, namely a catalog of domain-specific terminologies in use in environmental research, which will enable us to systematically compare existing resources for building the interoperability framework.

${ }^{1}$ https://www.rd-alliance.org/groups/interoperable-descriptions-observable-property-terminologywg-i-adopt-wg

${ }^{2}$ https://envri.eu/home-envri-fair/

${ }^{3}$ https://www.iso.org/standard/32574.html

${ }^{4}$ https://www.w3.org/TR/vocab-ssn/

${ }^{5}$ https://www.opengeospatial.org/standards/sensorml

${ }^{6}$ https://github.com/NCEAS/oboe/ 